\title{
A Power-Efficient Sensing Approach for Pulse Wave Palpation-Based Heart Rate Measurement
}

\author{
Gabriel Bravo, Jesús M. Silva, Salvador A. Noriega, Erwin A. Martínez (D), Francisco J. Enríquez \\ and Ernesto Sifuentes *(D)
}

check for

updates

Citation: Bravo, G.; Silva, J.M.;

Noriega, S.A.; Martínez, E.A.;

Enríquez, F.J.; Sifuentes, E. A

Power-Efficient Sensing Approach for

Pulse Wave Palpation-Based Heart

Rate Measurement. Sensors 2021, 21,

7549. https://doi.org/10.3390/

s21227549

Academic Editors: Paolo Viscont and Marc Parrilla

Received: 16 September 2021

Accepted: 10 November 2021

Published: 13 November 2021

Publisher's Note: MDPI stays neutral with regard to jurisdictional claims in published maps and institutional affiliations.

Copyright: (C) 2021 by the authors. Licensee MDPI, Basel, Switzerland. This article is an open access article distributed under the terms and conditions of the Creative Commons Attribution (CC BY) license (https:/ / creativecommons.org/licenses/by/ $4.0 /)$.
Institute of Engineering and Technology, Universidad Autónoma de Ciudad Juárez (UACJ), Ciudad Juárez 32310, Mexico; gbravo@uacj.mx (G.B.); jesilva@uacj.mx (J.M.S.); snoriega@uacj.mx (S.A.N.); emartine@uacj.mx (E.A.M.); fenrique@uacj.mx (F.J.E.)

* Correspondence: esifuent@uacj.mx; Tel.: +52-656-688-4841

\begin{abstract}
Heart rate (HR) is an essential indicator of health in the human body. It measures the number of times per minute that the heart contracts or beats. An irregular heartbeat can signify a severe health condition, so monitoring heart rate periodically can help prevent heart complications. This paper presents a novel wearable sensing approach for remote HR measurement by a compact resistance-to-microcontroller interface circuit. A heartbeat's signal can be detected by a Force Sensing Resistor (FSR) attached to the body near large arteries (such as the carotid or radial), which expand their area each time the heart expels blood to the body. Depending on how the sensor interfaces with the subject, the FSR changes its electrical resistance every time a pulse is detected. By placing the FSR in a direct interface circuit, those resistance variations can be measured directly by a microcontroller without using either analog processing stages or an analog-to-digital converter. In this kind of interface, the self-heating of the sensor is avoided, since the FSR does not require any voltage or bias current. The proposed system has a sampling rate of $50 \mathrm{Sa} / \mathrm{s}$, and an effective resolution of 10 bits $(200 \mathrm{~m} \Omega)$, enough for obtaining well-shaped cardiac signals and heart rate estimations in real time by the microcontroller. With this approach, the implementation of wearable systems in health monitoring applications is more feasible.
\end{abstract}

Keywords: wearable health monitoring; resistance-to-time interface circuit; force-sensing resistor; direct microcontroller interface circuit; heart rate measurement

\section{Introduction}

Health monitoring parameters (such as heart and respiration rate) measured by noninvasive sensing methods have been the object of study over the past decades [1]. In recent years, portable personal monitoring systems or wearable systems have been developed for monitoring the current state of a patient's health in everyday situations [2]. In the same way, methods for detecting physiological parameters have been designed by implementing monitoring systems in objects of daily use, such as a bed, a toilet seat, a computer mouse, or an electronic weight, to name a few. Furthermore, the integration of the Internet of Things (IoT) into healthcare has led to intelligent applications such as remote healthcare and intelligent healthcare monitoring systems.

Detecting cardiorespiratory signals using electromechanical sensors is a well-known technique used to assess a subject's health conditions. The mechanical reactions caused by heartbeats and respiration bring about low stress levels, which can be detected by force sensors attached to the body or placed in furniture, such as chairs, mattresses, and automobile security belts [2-10]. Formerly, Electromechanical Film (EMFi) and piezoelectric sensors were used; nowadays, Force-Sensing Resistors (FSRs) are preferred because of the simplicity of the interface circuits used to convert the resistance changes into output voltage or current [3]. FSRs are based on piezoresistive sensing technology, which has the advantage of providing an observable resistance change induced by minimal stresses. 
For this, Polymer Thick-Film FSRs have been implemented in wearable systems for ubiquitous and long-term monitoring, such as sitting posture recognition and muscle contraction, and to detect the cardiac activity from the foot sole [11-16].

To measure resistance changes in an FSR, it is common to use analog conditioning circuits that convert these changes into an output voltage. The most common alternative is to place the FSR into a voltage divider or Wheatstone bridge, the current excitation of which depends on the non-actuated resistance of the FSR [9]. In those circuits, the output voltage depends not only on the sensor's resistance change but also on the excitation voltage or current. Moreover, if the output voltage needed to suit the input range of an analog-to-digital converter (ADC), some analog signal processing stages would need to be implemented, such as amplification, level shifting, and filtering [17]. A more significant number of conditioning stages increases the power consumption and instrumentation size, which restricts the implementation of portable and low-power systems such as wearable wireless sensor nodes for health monitoring.

A simplified circuit to interface resistive sensors has been proposed and widely analyzed [18-21]. This circuit connects the sensors directly to a microcontroller (MCU) without using an analog signal conditioning path or ADC. In this topology, called Direct Interfaced Circuit (DIC), the MCU excites the resistive sensor to obtain a time-modulated signal digitized by a timer embedded into the MCU. Recently, DIC was used to obtain respiratory signals through a nasal thermistor [22] and by an FSR placed in the seat of a typical chair [16]. This paper presents a novel sensing approach for heart rate monitoring by a DIC (a single FSR connected directly to a low-cost MCU). The main benefits of this approach over traditional skin electrodes and optical sensors are: (1) analog signal conditioning stages can be omitted, which results in a compact and power-efficient HR system; (2) neither analog nor digital filters are needed due to the FSR-yielded, well-shaped, and noiseless heartbeat signal. Hence, HR can be estimated in real time by a simple algorithm implemented in the MCU without any additional digital signal processing. With this sensing approach, wearable systems for ubiquitous health telemonitoring are more feasible.

\section{Sensing Approach}

\subsection{Heartbeat Signal Detection}

FSR is a flexible, very low-cost, and robust polymer thick film (PTF) sensor whose resistance $\left(R_{\mathrm{s}}\right)$ changes with an increase in the force applied to the sensor's surface. Heartbeats can be detected by attaching the sensor to the skin in places near the big arteries, which expand their area each time the heart expels blood to the body [23]. Figure 1 depicts the proposed system for sensing the heartbeats of a subject; the FSR is placed near the radial artery with a Velcro wristband and changes its electrical resistance $\left(\Delta R_{\mathrm{s}}\right)$ every time a pulse is detected. Hence, if DIC is able to measure such small-resistance variations caused by cardiac activity, the HR of a subject can be measured. Moreover, the heartbeat's signal could be transmitted via Wi-Fi to a secured cloud server where any Wi-Fi device could access the report server with proper credential authentication.

\subsection{Direct Interface Circuit}

Figure 2a shows the circuit proposed to measure $R_{\mathrm{S}}$, which is the most basic direct sensor-to-MCU interface circuit [18]. The MCU only needs to have an embedded digital timer and two input/output (I/O) digital pins with external interruption capability. In summary, the DIC simultaneously performs a resistance-to-time conversion and a timeto-digital conversion, resulting in a digital number proportional to $T_{\text {dis }}$ and hence to $R_{\mathrm{s}}$. The MCU algorithm to obtain these measurements involves two stages: (a) charging stage and (b) discharging and time measurement stage (see Figure 2b). 


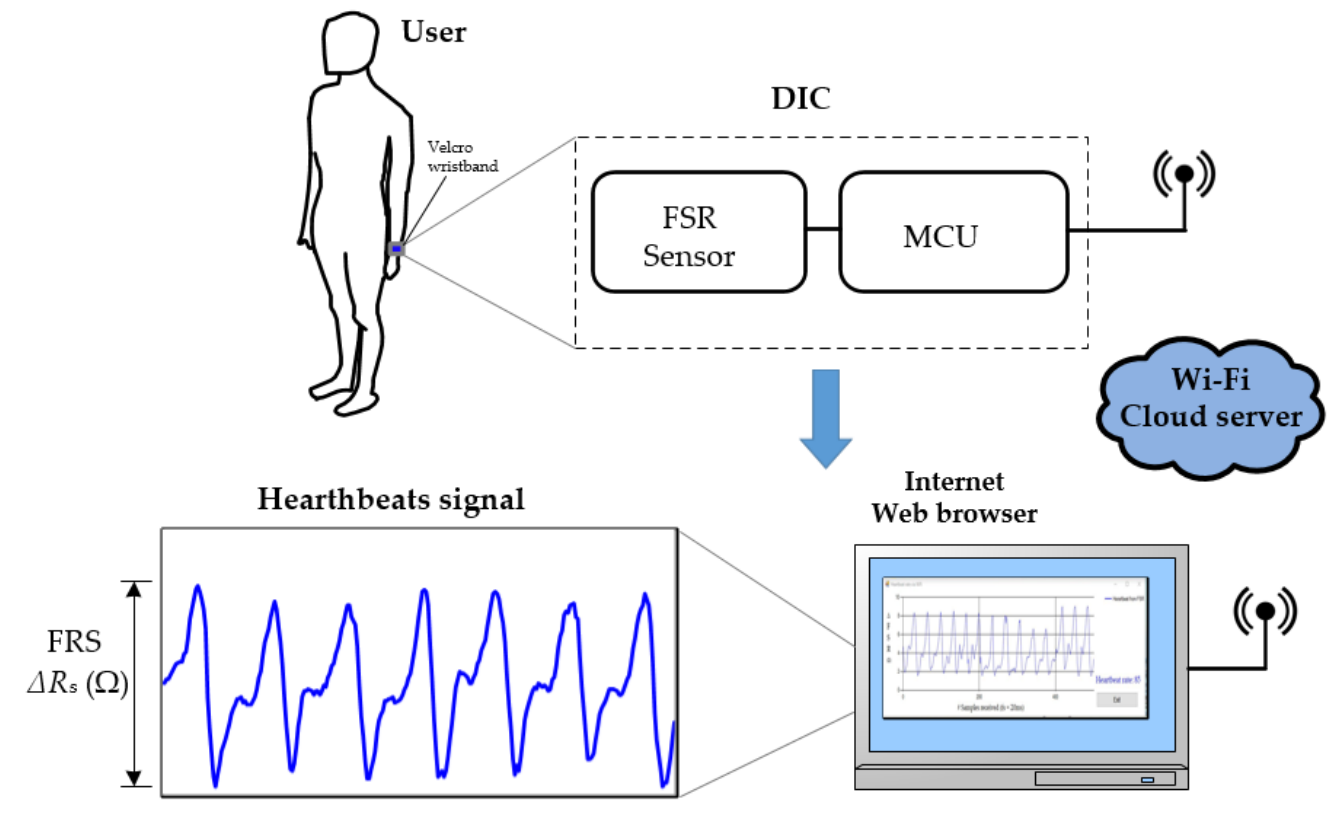

Figure 1. Wearable sensing approach for remote heart rate monitoring.

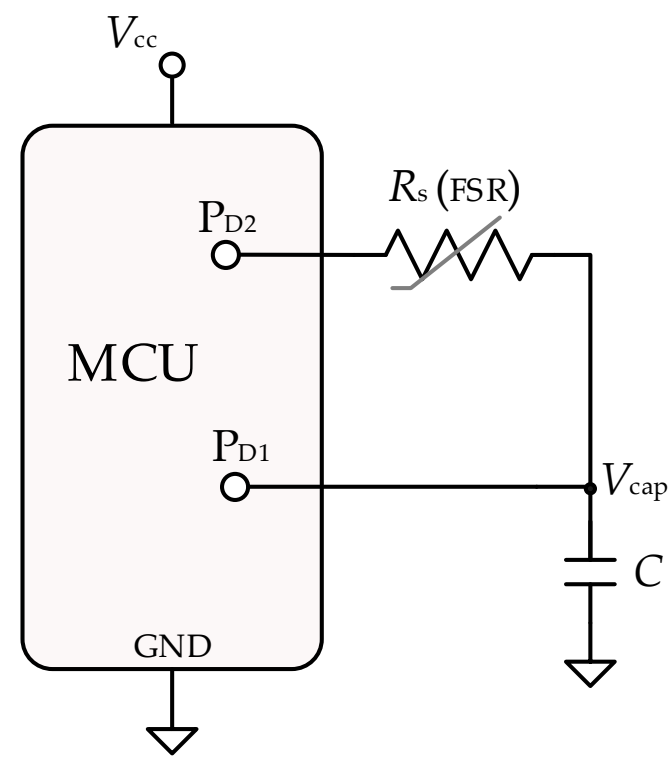

(a)

Figure 2. Cont. 


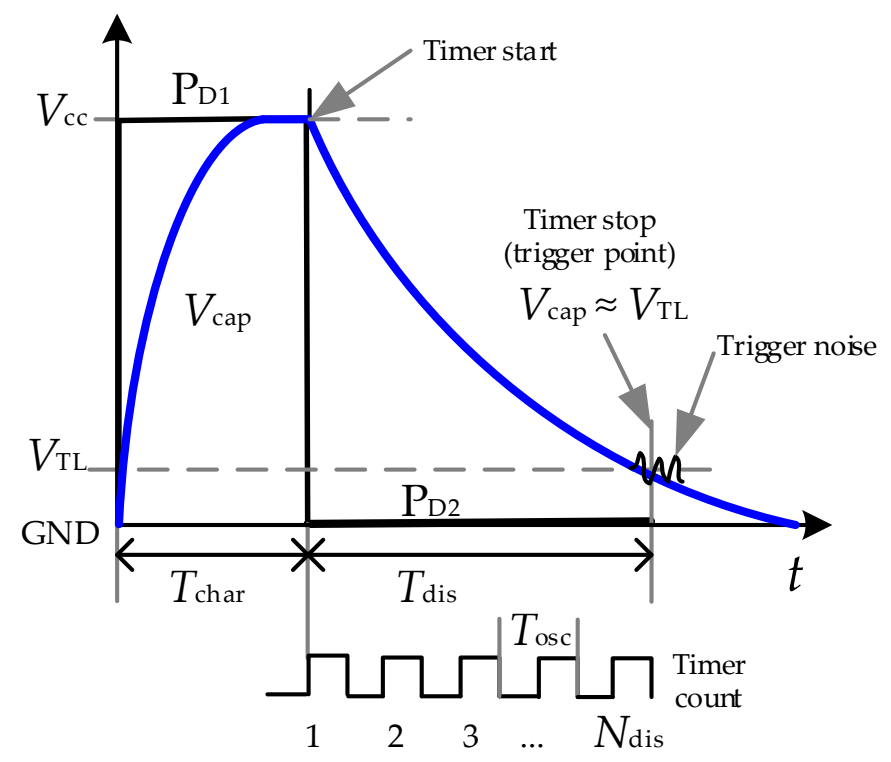

(b)

Figure 2. (a) DIC to measure the FSR variations $\left(\Delta R_{\mathrm{S}}\right)$ caused by heartbeats; (b) digital state of $\mathrm{P}_{\mathrm{D} 1}$ and $P_{\mathbf{D} 2}$ and the voltage across $C$ during each measurement of $R_{\mathbf{s}}$.

In the first stage, $P_{D 1}$ is set as an output providing a digital " 1 ", so $P_{D 1}$ generates a step pulse from GND to $V_{\text {cc }}$ (supply voltage of the MCU) or from " 0 " to " 1 " in the digital domain. At the same time, $\mathrm{P}_{\mathrm{D} 2}$ is set as an input, offering a high impedance state "HZ", so $C$ is charged towards $V_{\mathrm{cc}}$ through $R_{\mathrm{O} 1}$, with a time constant $\tau_{\mathrm{c}}=R_{\mathrm{O} 1} \mathrm{C}$. $R_{\mathrm{O} 1}$ represents the internal resistance of $P_{D 1}$, whose value is about tens of ohms [18], small enough to achieve a fast charging time. The charging stage must be at least $T_{\text {char }}=5 \tau_{\mathrm{c}}$ to ensure that $V_{\text {cap }}(t)$ reaches $V_{\text {cc. }}$. In the second stage, $\mathrm{P}_{\mathrm{D} 2}$ is set as an output providing a digital " 0 "; consequently, $C$ is discharged towards GND through $R_{\mathrm{x}}$, with a time constant $\tau_{\mathrm{d}}=R_{\mathrm{S}} C$, while the embedded timer of the MCU starts to measure the time interval required to do so. $\mathrm{P}_{\mathrm{D} 1}$ is configured as an input to interrupt the MCU on the falling edge. When $V_{\text {cap }} \approx V_{\mathrm{TL}}$ (low threshold voltage of the Schmitt Trigger (ST) buffer embedded into $\mathrm{P}_{\mathrm{D} 1}$ ), the ST triggers and the timer stops. The count of the timer $N_{\text {dis }}$ is the digital equivalent to the discharging time, $T_{\mathrm{dis}}$, proportional to $R_{\mathrm{S}}$ as:

$$
T_{\text {dis }}=N_{\text {dis }} T_{\mathrm{osc}}=R_{\mathrm{s}} C \ln \left(\frac{V_{\mathrm{CC}}}{V_{\mathrm{TL}}}\right)
$$

where $T_{\mathrm{osc}}$ is the period of the clock signal of the timer. $R_{\mathrm{s}}$ can be estimated from (1), assuming that $C, V_{\mathrm{cc}}$, and $V_{\mathrm{TL}}$ are known. The variability of these parameters can be compensated for by adding reference components and then applying auto-calibration techniques [18-20]. In this application, the DIC (Figure 2a) does not require any reference resistor because the information to be monitored (cardiac pulse signal) is contained in the change in $R_{\mathrm{S}}$, not in its absolute value, so the circuit's accuracy is less critical. On the other hand, the slew rate, $S R$, of the exponential discharging waveform at the trigger point is:

$$
S R=\frac{V_{\mathrm{TL}}}{R_{\mathrm{s}} \mathrm{C}}
$$

Ideally, the time-to-digital conversion has a resolution equal to $T_{\mathrm{osc}}$. However, the main uncertainty sources are the quantization of the timer and the trigger noise (the point 
when $V_{\text {cap }} \approx V_{\mathrm{TL}}$ and the timer stops is a noise-sensitive process) [24]. If only quantization effects are considered, the resolution of the DIC in bits, $M$, or ohms, $r$, respectively, is:

$$
\begin{gathered}
M=\operatorname{lb}\left(\frac{T_{\mathrm{dis}, \mathrm{max}}-T_{\mathrm{dis}, \mathrm{min}}}{T_{\mathrm{osc}}}\right) \\
r=\frac{R_{\mathrm{s}, \max }-R_{\mathrm{s}, \min }}{2^{M}}
\end{gathered}
$$

where $R_{\mathrm{s}, \min }$ and $R_{\mathrm{s}, \max }$ are the minimal and maximal resistance values that produce, respectively, the discharging times to be measured: $T_{\text {dis, min }}$ and $T_{\text {dis,max }}$.

From (1) and (3), a high resolution can be achieved by decreasing $T_{\text {osc }}$ or increasing $C$ (thus $\tau_{\mathrm{d}}$ ). However, a high value of $C$ increases the measuring time, thus reducing the bandwidth of the measurement system. So, a $C$ value that yields an optimal resolution must be selected [25]. By replacing (1) in (3) for a particular application requiring $M$ bits of resolution, the $C$ value should be:

$$
C \geq \frac{T_{\mathrm{osc}} 2^{M}}{\left(R_{\mathrm{s}, \max }-R_{\mathrm{s}, \min }\right) \ln \left(\frac{V_{\mathrm{CC}}}{V_{\mathrm{TL}}}\right)}
$$

On the other hand, the trigger noise superimposed on the exponential discharging voltage on $V_{\mathrm{TL}}$ limits the resolution of the measurement system to an effective number of bits (ENOB) lower than M. According to [24], the ENOB can be estimated by:

$$
\mathrm{ENOB} \approx M-\mathrm{lb}\left(\frac{\sqrt{u^{2}\left(T_{\mathrm{dis}, \max }\right)+u^{2}(z)}}{u(z)}\right)
$$

where $u\left(T_{\text {dis,max }}\right)$ is the standard uncertainty of $T_{\text {dis,max }}$ due to noise effects and $u(z)$ represents the quantization uncertainty that can be estimated as the standard deviation of the uniform distribution of width $T_{\mathrm{osc}}$, that is:

$$
u(z)=\frac{T_{\mathrm{osc}}}{\sqrt{12}}
$$

The variability of $T_{\text {dis }}$ can be analyzed by a statistical analysis of a series of observations. The histogram, the mean, and the standard deviation, $s\left(T_{\mathrm{dis}, \max }\right)$, permit us to characterize noise effects. So, $u\left(T_{\mathrm{dis}, \max }\right)$ from $k$ observations can be estimated by:

$$
u\left(T_{\mathrm{dis}, \max }\right)=\frac{s\left(T_{\mathrm{dis}}\right)}{\sqrt{k}}
$$

In summary, from (2), a small $R_{\mathrm{S}} C$ yields large $S R$, so quantization effects predominate over trigger noise, and the ENOB is equal to $M$. Conversely, a large $R_{\mathrm{S}} C$ implies slow $S R$, which makes the triggering process more susceptible to noise, increasing the count dispersion and the standard deviation $s\left(T_{\text {dis }}\right)$. So, there is a speed/resolution trade-off that depends mainly on $R_{\mathrm{s}} C$. By establishing a measurement range, $R_{\mathrm{s}, \max }-R_{\mathrm{s}, \min }$, in a particular application to achieve $M$ bits of resolution, equation (5) can be used as a first design rule to estimate $C$.

\section{Materials and Method}

The DIC (Figure 2a) was implemented by a D1 mini ESP32 module. This module was designed for mobile electronics and Internet-of-Things (IoT) applications. It has an MCU (ESP32 from Espressif Systems) that contains Wi-Fi and Bluetooth (BLE embedded) and $520 \mathrm{kB}$ of RAM. The module was powered by a rechargeable battery $5 \mathrm{~V}$ at $1 \mathrm{~A}$ and $2.2 \mathrm{Ah}$, and the MCU with $V_{\mathrm{cc}}=3.3 \mathrm{~V}$ by a voltage regulator incorporated in the module. $R_{\mathrm{S}}$ was an FSR-402 sensor (from Interlink Electronics) with a sensible area of $14.68 \mathrm{~mm}$ in 
diameter, a nominal thickness of $0.46 \mathrm{~mm}, R_{\mathrm{s}}>10 \mathrm{M} \Omega$ without force applied, and rise time of $<3 \mu$ s. To know the sensor response, known forces from $1 \mathrm{~N}$ to $16.5 \mathrm{~N}$ were applied (Figure 3). In addition, the nominal $R_{\mathrm{S}}$ value was measured with a digital multimeter when the FSR was placed in a Velcro wristband near the radial artery, the nominal value was $R_{\mathrm{S}}=R_{\mathrm{s}, 0} \approx 5 \mathrm{k} \Omega$.

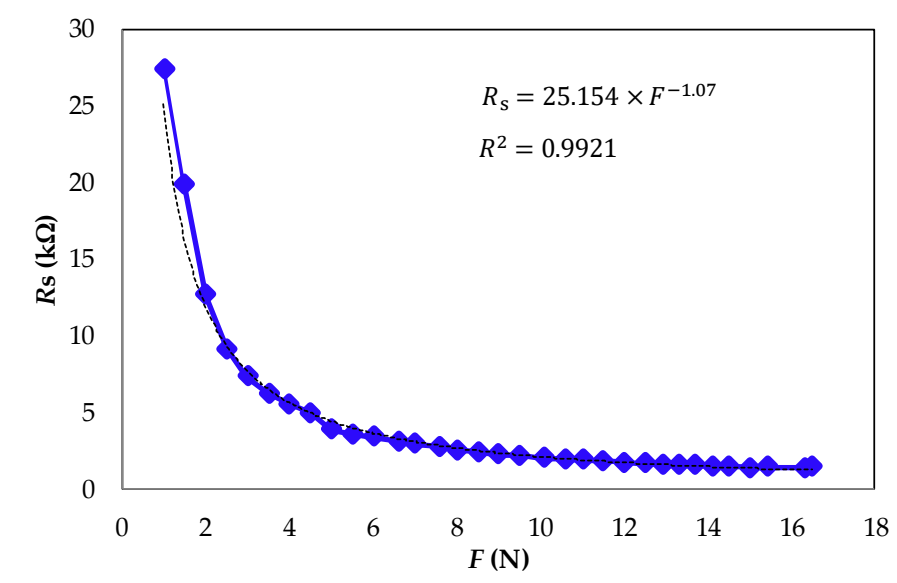

Figure 3. Experimental response of the FSR-402 when force is applied to the sensor's surface.

The MCU program to measure $T_{\text {dis }}$ (and so $R_{\mathrm{s}}$ ) by following the sequence described in Section 2.2 was implemented in $C$ language. $P_{D 1}$ and $P_{D 2}$ (Figure 2a) were implemented by GPIOs 13 and 14, respectively. (The MCU works at $80 \mathrm{MHz}$, so the digital timer measures $T_{\text {dis }}$ with a resolution of $T_{\text {osc }}=12.5 \mathrm{~ns}$.) From each measurement of $T_{\text {dis }}, R_{\mathrm{s}}$ was obtained from (1). The MCU parameters $V_{\mathrm{cc}}=3.23 \mathrm{~V}, V_{\mathrm{TL}}=1.56 \mathrm{~V}, R_{\mathrm{O} 1}=32 \Omega$ were obtained from the procedures described in [18]. By considering $\Delta R_{\mathrm{s}, \max }=R_{\mathrm{s}, \max }-R_{\mathrm{s}, \min } \approx 200 \Omega$ and $M=10$ bits, from (5), the minimal value of $C$ is $86 \mathrm{nF}$, a metallized polypropylene with $C$ $=150 \mathrm{nF}$ was chosen. According to [25], a DIC with these values would be capable of a maximum sampling rate of $1.75 \mathrm{kSa} / \mathrm{s}$, more than enough for this application.

An adult's regular resting HR ranges from 60-100 BPM, i.e., between approximately 1 and 2 beats per second. For a reasonable reconstruction of the heartbeat signal, a sampling rate of $S_{f}=50 \mathrm{Sa} / \mathrm{s}$ was selected, which was configured and controlled by an internal digital timer. Hence, the DIC takes a sample of $R_{\mathrm{s}}$ every $1 / S_{f}=20 \mathrm{~ms}$ and stores it in its RAM. The acquisition time for $N_{\mathrm{s}}$ samples was defined as $T_{\mathrm{acq}}=N_{\mathrm{s}}\left(1 / S_{f}\right)$. For concurrent records of $10 \mathrm{~s}$ (of heartbeat signal), the MCU was programmed to measure and save (in RAM) 500 samples of $R_{\mathrm{s}}$. Simultaneously, the MCU calculates the heart rate from the samples in real time by taking the period from two consecutive slopes, based on the abrupt downward slope shown in each beat of the signal (see Figure 4c). Each record of 500 samples and heart rate value was sent via Wi-Fi to a secured cloud server.

The sensing approach for remote heart rate monitoring was experimentally tested with five volunteers. Volunteers were asked to remain quiet during the measurement (10 s approximately) to avoid motion artifacts caused by hand movements. The FSR was attached with a Velcro band directly on the skin near the radial artery, as shown in Figure $4 \mathrm{~b}$. A commercial heart rate monitor (pulse sensor from World Famous Electronics) was used as a comparison to test the feasibility of the proposed system. This device combines a simple optical heart rate sensor with amplification and noise cancellation circuitry, making it fast and easy to obtain reliable pulse readings. Both signals were acquired by the same MCU: the FSR signal was acquired by the DIC interface algorithm (blue curve in Figure 4c), and the signals from the commercial monitor by the embedded ADC (green curve in Figure 4c). The graphical visualization of both signals was realized in a PC program implemented in Visual $\mathrm{C}^{\mathrm{TM}}$. The communication between the PC and the prototype for this test was via Wi-Fi and USB. 


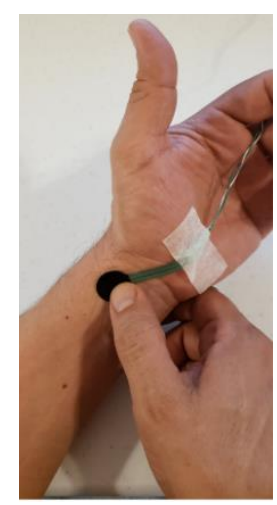

(a)

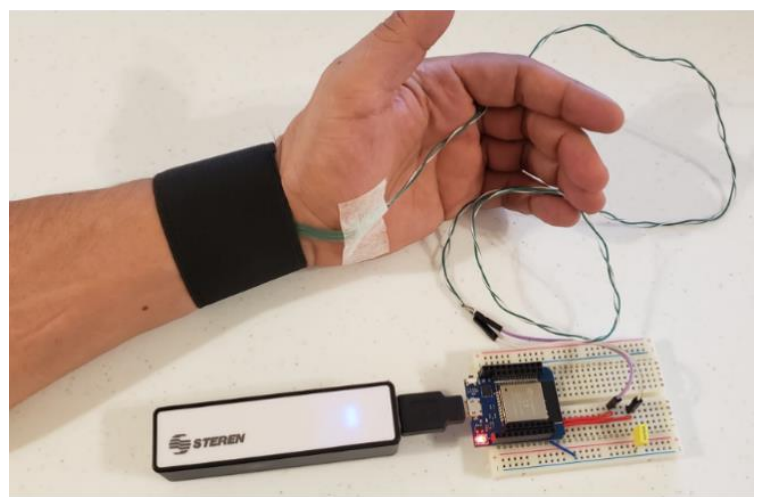

(b)

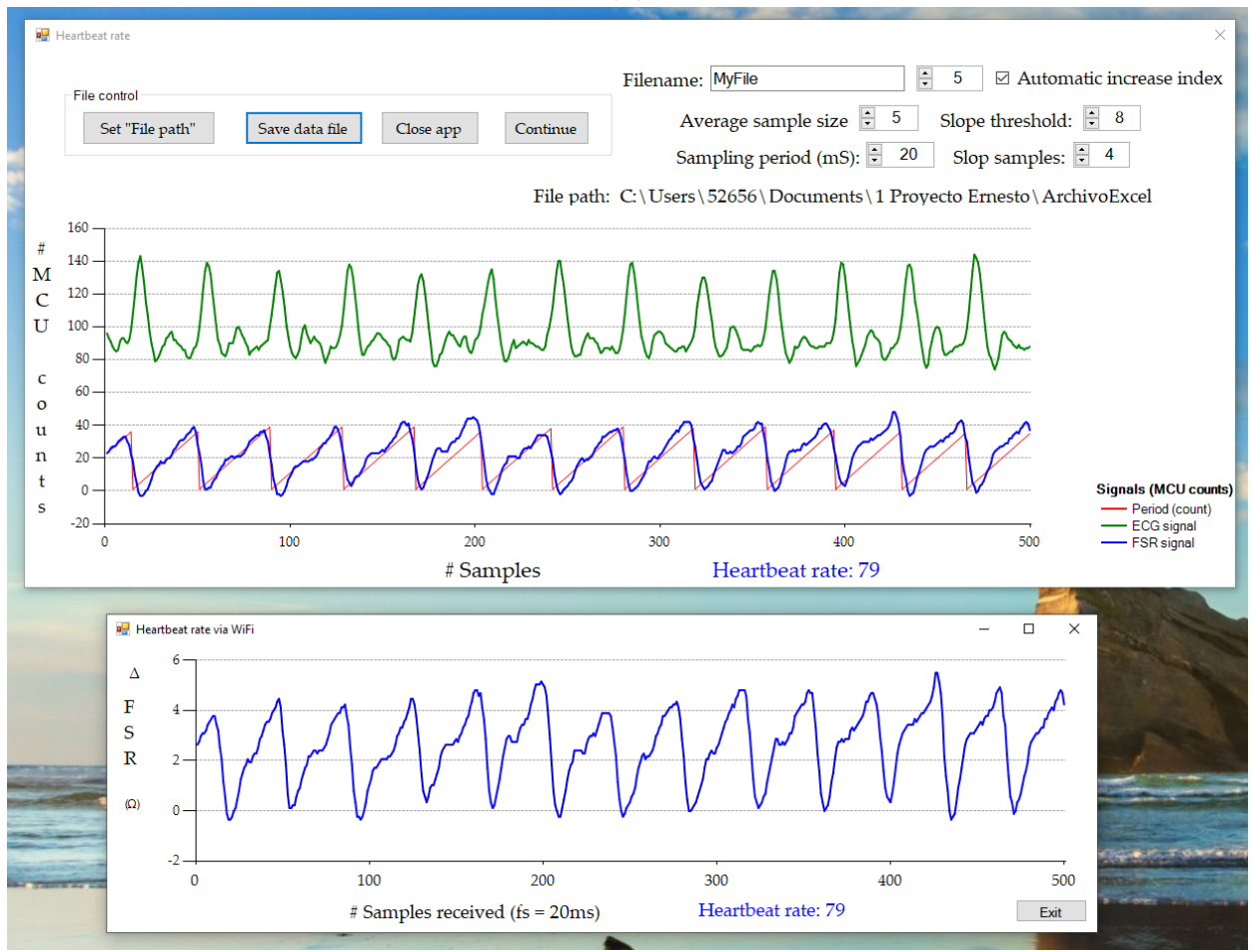

(c)

Figure 4. Prototype of the wearable sensing approach for remote heart rate monitoring. (a) FSR placed over the radial artery; (b) FSR with a Velcro wristband connected to a DIC; (c) graphical interface for remote visualization of the heartbeat signal and HR value.

We used the Bland-Altman plot to examine the agreement between the proposed wearable system and commercial heart rate monitor. Ten records of heartbeat signals 
were taken from each volunteer. As proposed in [26,27], the Limit of Agreement (LoA) was obtained with a $95 \%$ confidence interval as $[\mu-1.96 \sigma, \mu+1.96 \sigma]$, where $\mu$ is the average difference, and $\sigma$ is the standard deviation. Pearson's correlation between the two measurement systems was also estimated.

\section{Experimental Results and Discussion}

Figure 3 shows the experimental response of the FSR-402 obtained from the characterization. It shows how the resistance $\left(R_{\mathrm{S}}\right)$ decreases nonlinearly as force is applied to the sensor's surface. Nonlinearity errors could modify the shape of the heartbeat signal but not the HR estimation. Figure 4 depicts the wearable sensing approach for remote heart rate monitoring: (a) FSR-402 placed directly on the skin, (b) FSR with a Velcro wristband near the radial artery connected to a DIC, and (c) remote visualization of the heartbeats signal and HR value.

Figure 5 shows how the MCU reconstructs the heartbeat signal by a sampling rate of $50 \mathrm{kSa} / \mathrm{s}$ and 500 samples (a record of $10 \mathrm{~s}$ ). Simultaneously, the MCU calculates the HR by taking the period from two consecutive abrupt slopes, beat per beat of the signal (Sawtooth signal). In this case, HR was 79 BPM (see Figure 4c).

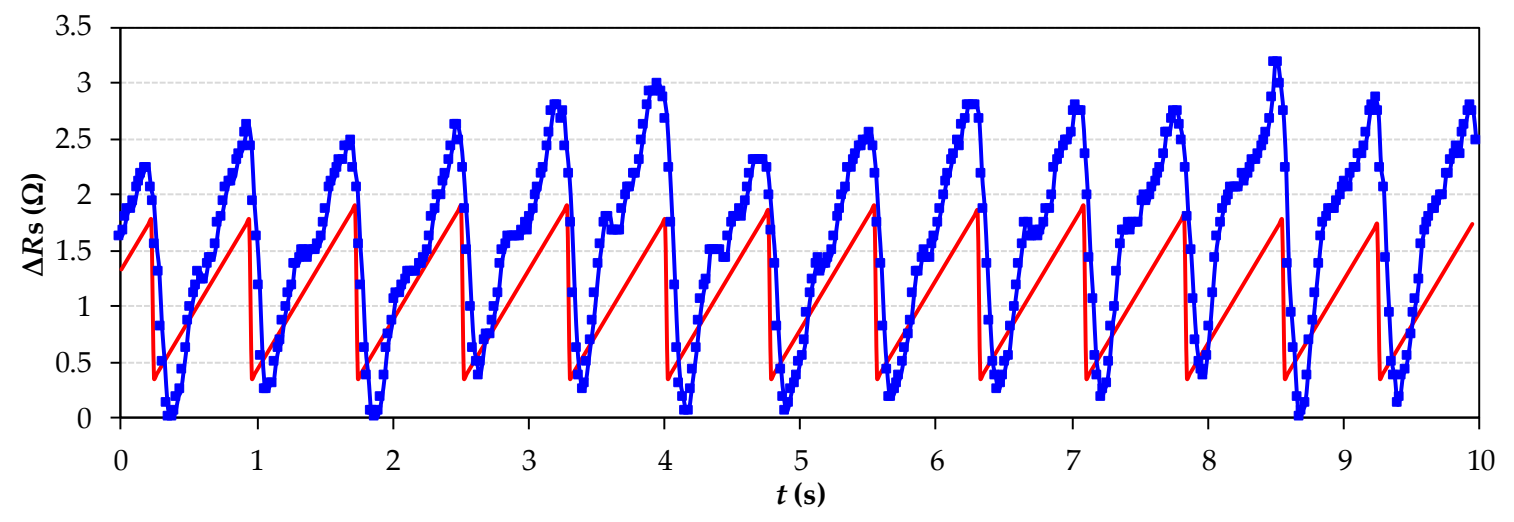

Figure 5. Experimental heartbeat signal reconstruction and HR measurement by the downward slope shown in each signal beat (the MCU implements the algorithm in real time).

Figures 6a and 7a show the resistance variations $\Delta R_{\mathrm{s}}$ of the FSR measured by the DIC (Figure 2a) of volunteers S1 and S2, respectively. In the same way, Figures $6 \mathrm{~b}$ and $7 \mathrm{~b}$ show the voltage variations, $\Delta V$, of the commercial $\mathrm{HR}$ monitor. Comparing the signals, a peak resistance change on $R_{\mathrm{S}}$ occurs after a QRS complex, implying that the signal registered with the DIC is related to cardiac activity. The heartbeat signal obtained from the FSR system is consistently compared with that obtained from the commercial monitor. In all cases, both signals match the number of beats. The FSR changes its electrical resistance each time a pulse is detected, and this pulse rate refers to the contraction and expansion of the artery when blood passes. Each time the heart expels blood into the body, the radial artery expands its area, applying pressure on the FSR and causing a downward change in the resistance of the FSR. Consequently, because the FSR is a sensor with a negative force coefficient, the shape obtained by the FSR corresponds to an inverted cardiac pulse signal. Since this is the natural response of the FSR sensor and does not affect the heart rate estimation, nothing further was performed with this result. As a result, the DIC was able to detect resistance changes caused by cardiac activity, enough to obtain well-shaped heartbeat signals and HR estimation. These were small-signal resistance variations of around $\pm 3.5 \Omega$ (for S1) and $\pm 1.5 \Omega$ (for S2). On the other hand, the sensitivity of the proposed method relies on the sensor sensitivity. Since the FSR is not supplied by any constant voltage or current, self-heating problems are avoided, and the sensitivity does not depend on any polarization source, as usually happens in conventional signal conditioning systems. 


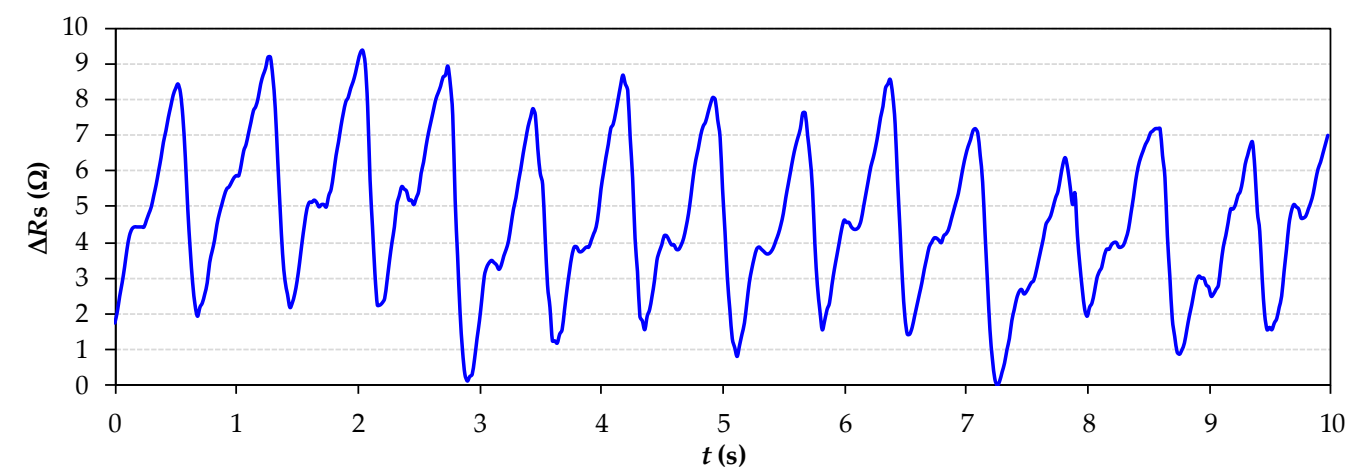

(a)

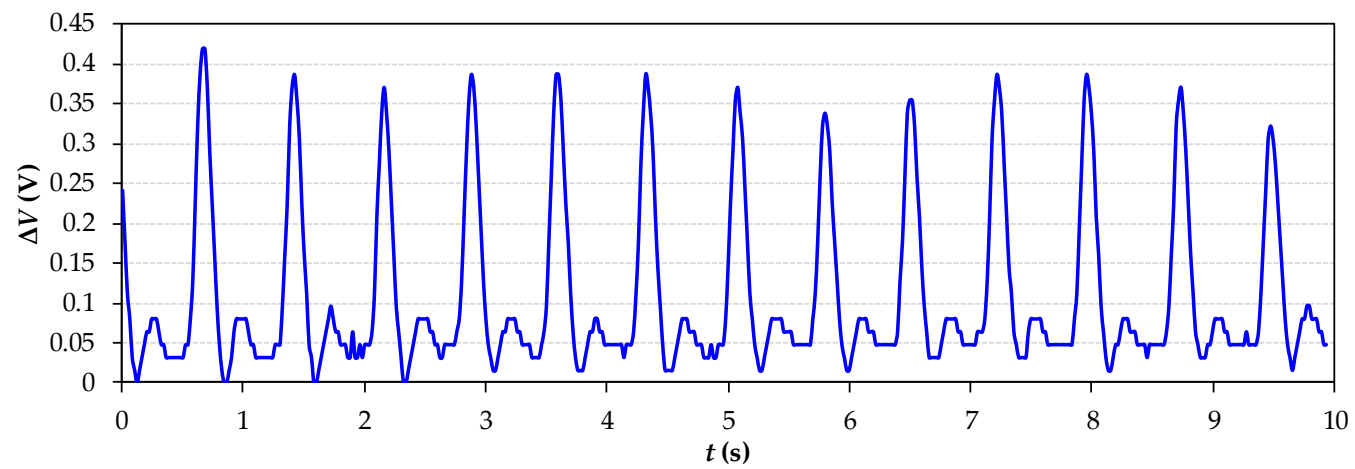

(b)

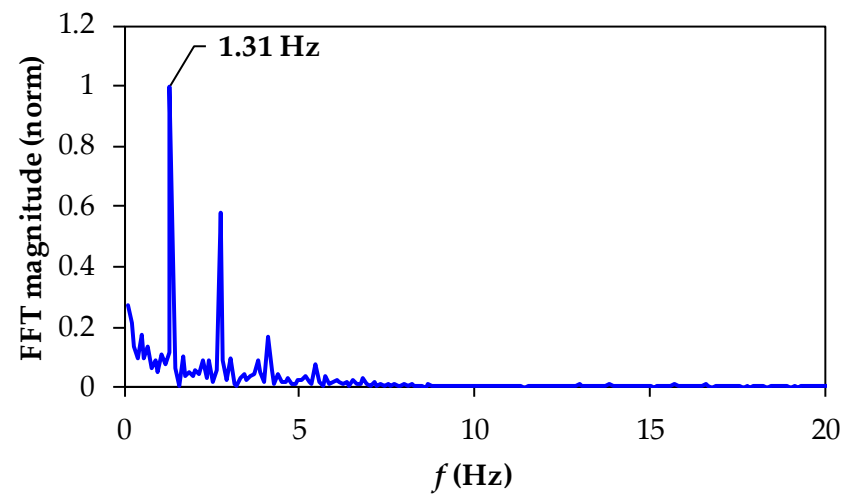

(c)

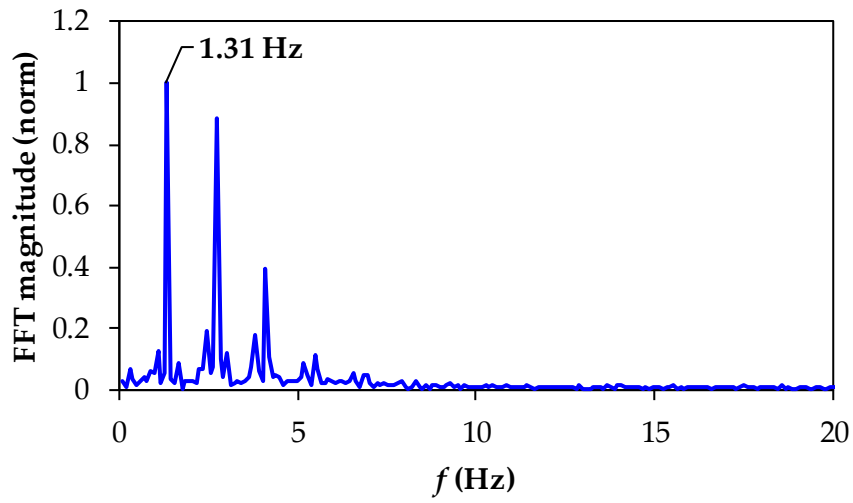

(d)

Figure 6. Experimental heartbeat signal from S1. (a) Resistance variations in FSR measured by the DIC when the sensor was located near the radial artery; (b) voltage variations in the commercial heart rate monitor; (c) frequency spectrum of the FSR signal; (d) frequency spectrum of the commercial monitor signal. 


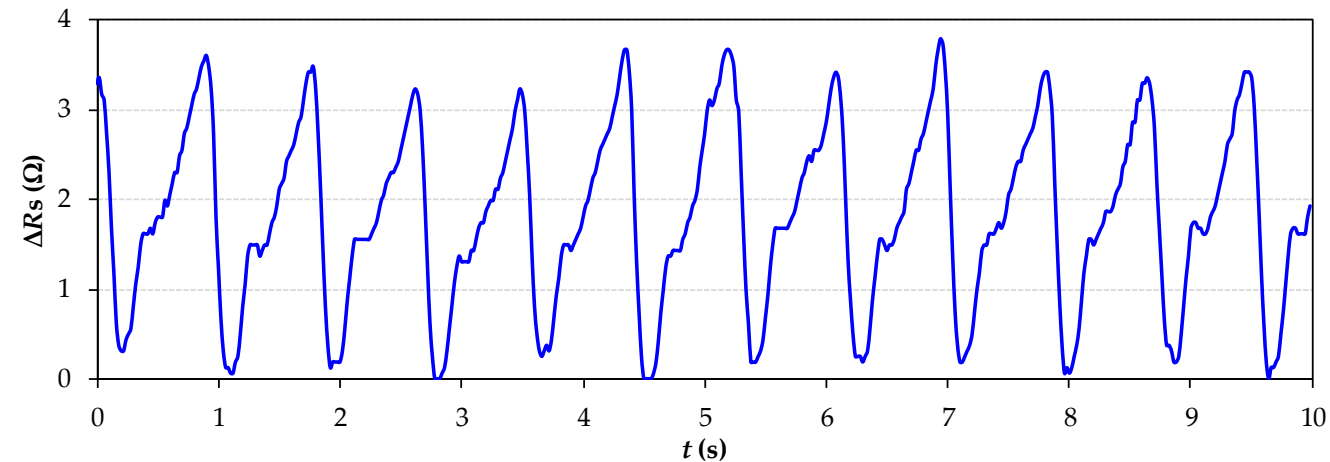

(a)

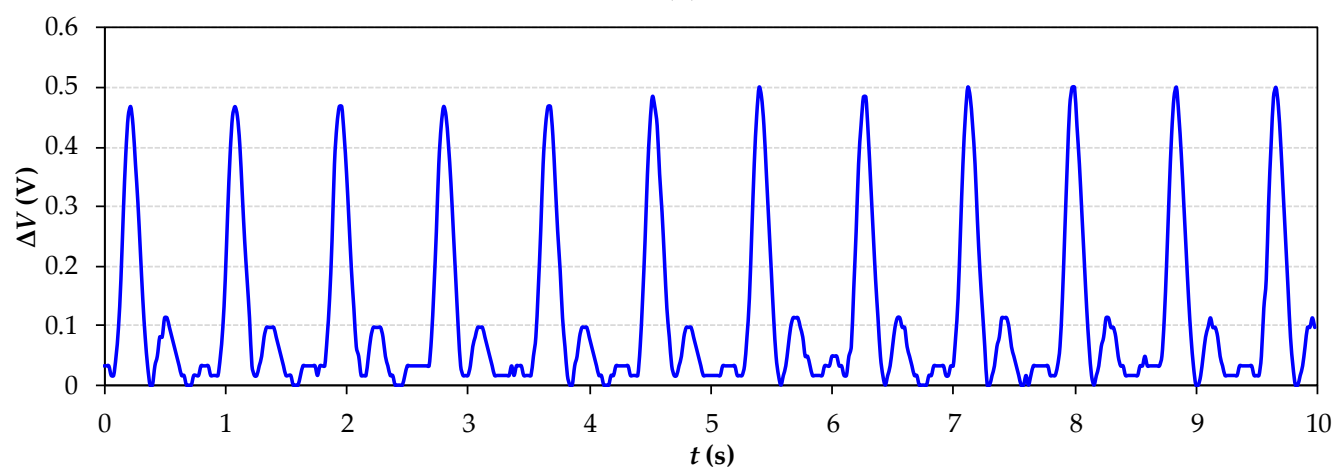

(b)

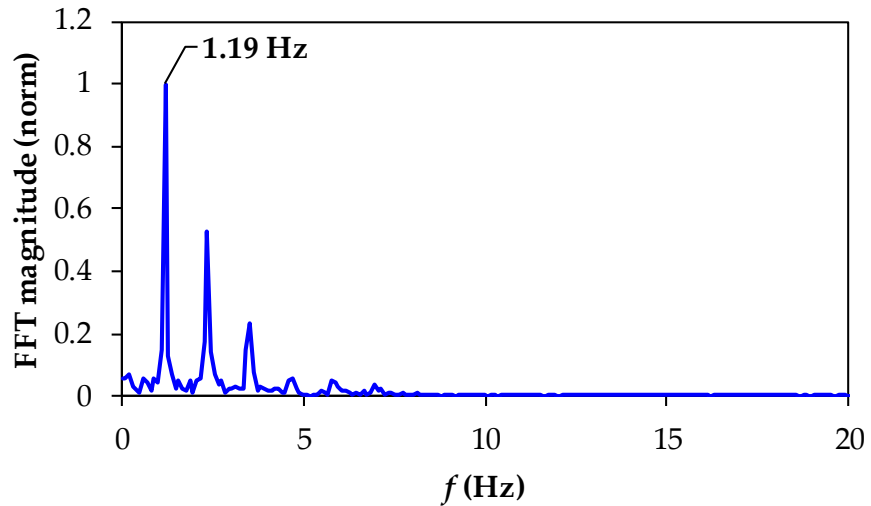

(c)

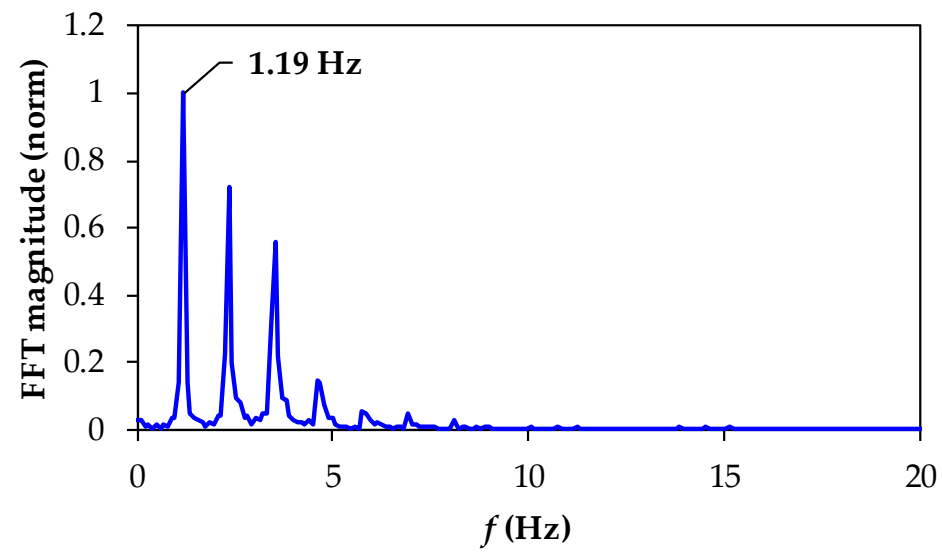

(d)

Figure 7. Experimental heartbeat signal from S2. (a) Resistance variations in FSR measured by the DIC when the sensor was located near the radial artery; (b) voltage variations in the commercial heart rate monitor; (c) frequency spectrum of the FSR signal; (d) frequency spectrum of the commercial monitor signal. 
Figure $6 c$,d show the frequency spectrum of the heartbeat signal for S1; in both figures, there was a fundamental peak at $1.31 \mathrm{~Hz}$ (79 BPM). In the same way, Figure $7 \mathrm{c}$, d show the frequency spectrum of the heartbeat signals for S2; in both cases, there was a fundamental peak at $1.19 \mathrm{~Hz}$ (72 BPM). These results demonstrate that the proposed wearable system is able to detect cardiac activity beat by beat, and it is also able to detect different heart rates. Furthermore, the FSR signals have a negligible contribution of noise, which facilitates the estimation of HR.

The Bland-Altman plot that compares the heartbeat signals (peak to peak) measured from the five volunteers (500 records) by the DIC and by the commercial monitor is shown in Figure 8. The average difference, the standard deviation, and LoA were, respectively: $\mu=0.018, \sigma=2.28$, and [-4.45, 4.49] all in BPM. The histogram in Figure 9 shows a normal distribution of the peak-to-peak differences from the HR measured by DIC and by commercial monitor. As observed in the Bland-Altman plot, from $60 \mathrm{BPM}$ to approximately $96 \mathrm{BPM}$ there is a high agreement between the two measurement systems. The scatter plot between the HR measured by the DIC and by commercial monitor is given in Figure 10. The Pearson coefficient was 0.9817 , which is a statistically significant correlation. Therefore, it is shown that there is an excellent coincidence between the signals obtained with the DIC and the commercial system.

A summary of wrist-type PPG methods for HR monitoring is presented in Tables 4 and 5 from [28]. To compare our proposed sensing approach for HR measurement, we selected those techniques whose performance was evaluated by Bland-Altman analysis and Pearson's coefficient. The experimental performance of our system (LoA: [-4.45, 4.49] BPM, $\sigma=2.28 \mathrm{BPM}$, and Pearson coefficient of 0.9817 ) yielded similar results to six of the eleven methods presented in Tables 4 and 5 from [28].

As is well known, the presence of motion artifacts (MA) in the analysis and interpretation of cardiovascular signals, obtained by different methods ranging from mechanical to electrical and optical systems, is inevitable [29]. This work aims to estimate the HR of subjects in a quasi-stationary position avoiding MA caused by hand movement during the measurement (10 s approximately). As shown in Figures 6 and 7, the FSR signals measured by the DIC when the sensor was located near the radial artery have a negligible noise contribution, which facilitates the estimation of HR. The experimental results prove that it is possible to estimate the HR in real time using a DIC without any additional digital signal processing.

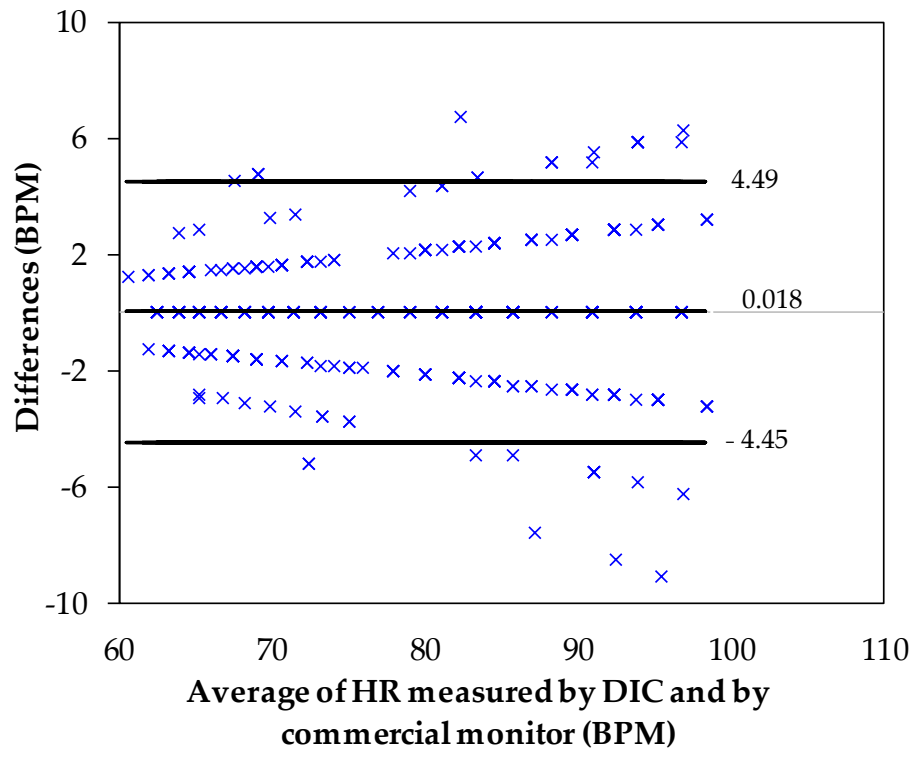

Figure 8. Bland-Altman plot that compares the HR measured by DIC and by the commercial monitor peak to peak. 


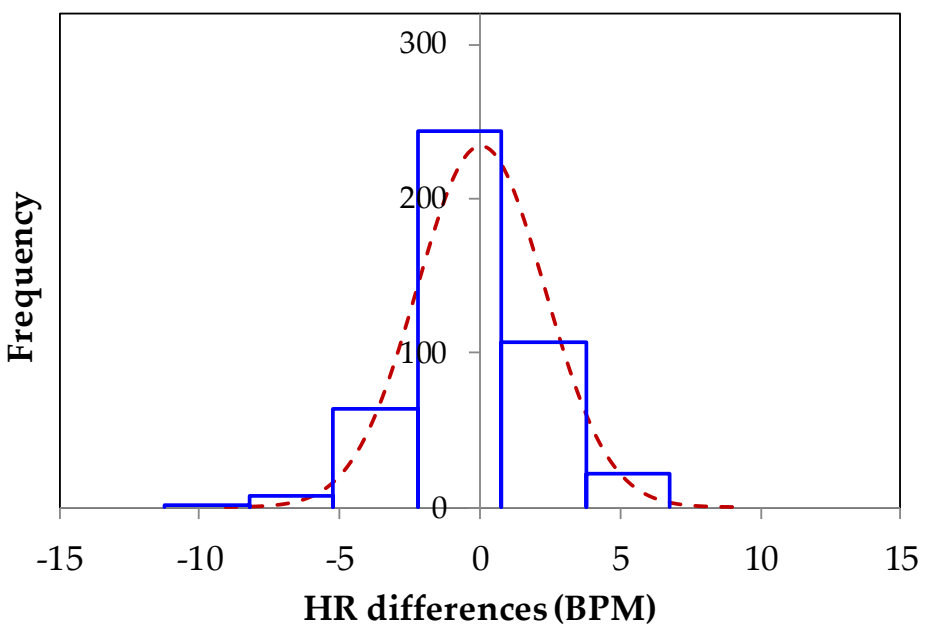

Figure 9. Histogram of the peak-to-peak differences between the HR measured by the DIC and by the commercial monitor.

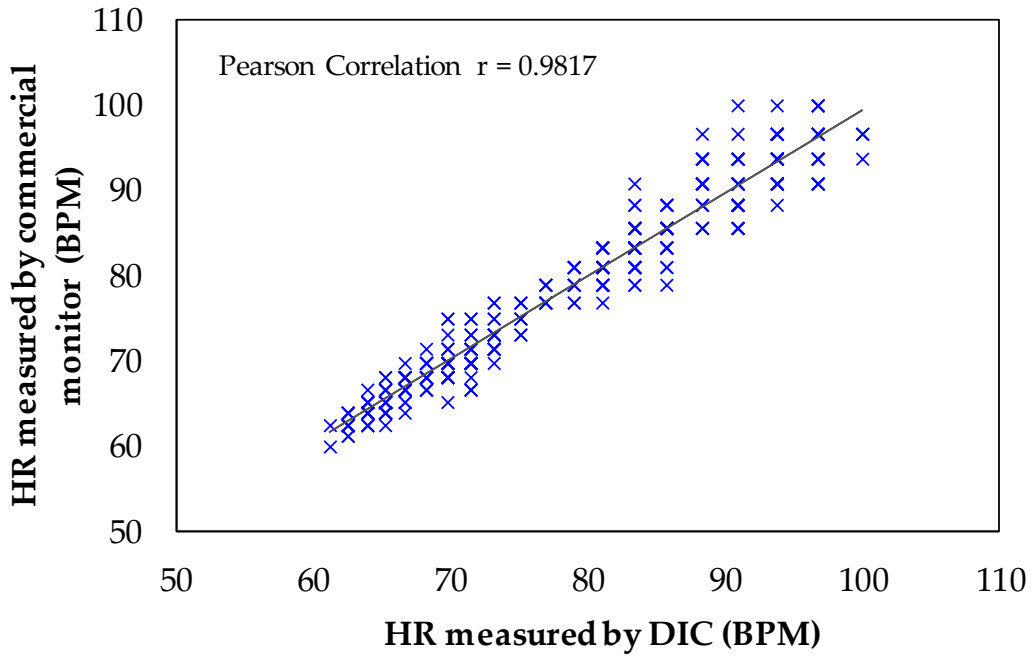

Figure 10. Scatter plot of the peak-to-peak HR measured by the DIC and by the commercial monitor.

\section{Conclusions}

A force-sensing resistor, such as an FSR-402, placed in a wristband near the radial artery and connected directly to a low-cost MCU yields a reliable, compact, and low-cost heart rate monitor. The experimental results show that small-resistance variations caused by the heartbeats of a subject bring well-shaped heartbeat signals from which it is possible to estimate the HR in real time by a simple MCU algorithm without any additional digital signal processing. Using this approach, wearable systems or wireless sensor nodes for health monitoring with high power efficiency are feasible.

Author Contributions: G.B. and J.M.S. proposed the main idea and, together with E.S., F.J.E., S.A.N. and E.A.M. built the measurement setup, performed the experiments, and analyzed the data. E.S., G.B., J.M.S. and F.J.E. interpreted the results and wrote the manuscript. All authors have read and agreed to the published version of the manuscript.

Funding: This research was funded by PRODEP and UACJ, México.

Institutional Review Board Statement: The study was conducted according to the guidelines of the Declaration of Helsinki, and approved by Research Ethics Committee of Universidad Autónoma de Ciudad Juárez (protocol number CEI-2021-2-63 approved on 24 September 2021). 
Informed Consent Statement: Informed consent was obtained from all subjects involved in the study.

Data Availability Statement: Not applicable.

Conflicts of Interest: The authors declare no conflict of interest.

\section{References}

1. Brüser, C.; Antink, C.H.; Wartzek, T.; Walter, M.; Leonhardt, S. Ambient and Unobtrusive Cardiorespiratory Monitoring Techniques. Biomed. Eng. IEEE Rev. 2015, 8, 30-43. [CrossRef]

2. Ding, X.; Clifton, D.; Ji, N.; Lovell, N.H.; Bonato, P.; Chen, W.; Zhang, Y.T. Wearable sensing and telehealth technology with potential applications in the coronavirus pandemic. IEEE Rev. Biomed. Eng. 2020, 14, 48-70. [CrossRef] [PubMed]

3. Siivola, J. New noninvasive piezoelectric transducer for recording of respiration, heart rate and body movements. Med. Biol. Eng. Comput. 1989, 27, 423-424. [CrossRef] [PubMed]

4. Koivistoinen, T.; Junnila, S.; Värri, A.; Kööbi, T. A New Method for Measuring the Ballistocardiogram using EMFi Sensors in a Normal Chair. In Proceedings of the 26th Annual International Conference of the IEEE EMBS, San Francisco, CA, USA, 1-5 September 2004; pp. 2026-2029.

5. Lin, Y.; Leng, H.; Yang, G.; Cai, H. An intelligent noninvasive sensor for driver pulse wave measurement. Sens. J. IEEE 2007, 7, 790-799. [CrossRef]

6. Griffiths, E.; Scott, T.; Bernheim, A.J. Health chair: Implicitly sensing heart and respiratory rate. In Proceedings of the 2014 ACM International Joint Conference on Pervasive and Ubiquitous Computing, Seattle, WA, USA, 13-17 September 2014 ; pp. 661-671.

7. Hamdani, S.T.A.; Fernando, A. The Application of a Piezo-Resistive Cardiorespiratory Sensor System in a Automobile Safety Belt. Sensors 2015, 15, 7742-7753. [CrossRef] [PubMed]

8. Wang, D.; Zhang, D.; LU, G. A Novel Multichannel Wrist Pulse System With Different Sensor Arrays. Instrum. Meas. IEEE Trans. 2015, 64, 2020-2034. [CrossRef]

9. Yaniger, S.I. Force sensing resistors: A review of the technology. In Proceedings of the Electro International IEEE, New York, NY, USA, 16-18 April 1991; pp. 666-668.

10. Samaun, S.; Wise, K.; Nielsen, E.; Angell, J. An IC piezoresistive pressure sensor for biomedical instrumentation. Biomed. Eng. IEEE Trans. 1973, 2, 101-109. [CrossRef] [PubMed]

11. Paradiso, R.; Loriga, G.; Taccini, N. A wearable health care system based on knitted integrated sensors. Inf. Technol. Biomed. 2005, 9, 337-344. [CrossRef]

12. Taccini, N.; Loriga, G.; Pacelli, M.; Paradiso, R. Wearable monitoring system for chronic cardio-respiratory diseases. In Proceedings of the Engineering in Medicine and Biology Society, EMBS, Vancouver, Canada, 20-25 August 2008; pp. 3690-3693.

13. $\mathrm{Hu}, \mathrm{Q} . ;$ Tang, X.; Tang, W. A smart chair sitting posture recognition system using flex sensors and FPGA implemented artificial neural network. IEEE Sens. J. 2020, 20, 8007-8016. [CrossRef]

14. Morales, I.; González-Landaeta, R.; Simini, F. Pressure sensors used as bioimpedance plantar electrodes: A feasibility study. In Proceedings of the 2021 IEEE International Symposium on Medical Measurements and Applications (MeMeA), Lausanne, Switzerland, 23-25 June 2021; pp. 1-6.

15. Esposito, D.; Andreozzi, E.; Fratini, A.; Gargiulo, G.D.; Savino, S.; Niola, V.; Bifulco, P. A piezoresistive sensor to measure muscle contraction and mechanomyography. Sensors 2018, 18, 2553. [CrossRef] [PubMed]

16. Sifuentes, E.; Gonzalez-Landaeta, R.; Cota-Ruiz, J.; Reverter, F. Seat occupancy detection based on a low-power microcontroller and a single FSR. Sensors 2019, 19, 699. [CrossRef] [PubMed]

17. Pallàs-Areny, R.; Webster, J.G. Sensors and Signal Conditioning, 2nd ed.; John Wiley \& Sons: New York, NY, USA, $2001 ;$ pp. 94-109.

18. Reverter, F.; Pallàs-Areny, R. Direct Sensor-to-Microcontroller Interface Circuits, 1st ed.; Marcombo: Barcelona, Spain, 2005; pp. 94-109.

19. Reverter, F. The art of directly interfacing sensors to microcontrollers. Low Power Electron. Appl. J. 2012, 2, 265-281. [CrossRef]

20. Reverter, F. Direct interface circuits for sensors. In Smart Sensors and MEMS: Intelligent Devices and Microsystems for Industrial Applications, 1st ed.; Nihtianov, S., Luque, A., Eds.; Woodhead Publishing: Sawston, Cambridge, UK, $2014 ;$ pp. $27-62$.

21. Anandanatarajan, R.; Mangalanathan, U.; Gandhi, U. Enhanced microcontroller interface of resistive sensors through resistanceto-time converter. IEEE Trans. Instrum. Meas. 2019, 69, 2698-2706. [CrossRef]

22. Sifuentes, E.; Cota-Ruiz, J.; González-Landaeta, R. Respiratory Rate Detection by a Time-Based Measurement System. Rev. Mex. De Ing. Bioméd. 2016, 37, 91-99.

23. Shu, Y.; Li, C.; Wang, Z.; Mi, W. A Pressure Sensing System for Heart Rate Monitoring with Polymer-Based Pressure Sensors and an Anti-Interference Post Processing Circuit. Sensors 2015, 15, 3224-3235. [CrossRef]

24. Reverter, F.; Pallàs-Areny, R. Effective number of resolution bits in direct sensor-to-microcontroller interfaces. Meas. Sci. Technol. 2004, 15, 2157-2162. [CrossRef]

25. Sifuentes, E.; Gonzalez-Landaeta, R.; Cota-Ruiz, J.; Reverter, F. Measuring dynamic signals with direct sensor-to-microcontroller interfaces applied to a magnetoresistive sensor. Sensors 2017, 17, 1150. [CrossRef] [PubMed]

26. Bland, J.M.; Altman, D. Statistical methods for assessing agreement between two methods of clinical measurement. Lancet 1986, 327, 307-310. [CrossRef] 
27. Bland, J.M.; Altman, D.G. Agreement between methods of measurement with multiple observations per individual. J. Biopharm. Stat. 2007, 17, 571-582. [CrossRef] [PubMed]

28. Nie, L.; Berckmans, D.; Wang, C.; Li, B. Is continuous heart rate monitoring of livestock a dream or is it realistic? A review. Sensors 2020, 20, 2291. [CrossRef]

29. Lee, J.; Kim, M.; Park, H.-K.; Kim, I.Y. Motion Artifact Reduction in Wearable Photoplethysmography Based on Multi-Channel Sensors with Multiple Wavelengths. Sensors 2020, 20, 1493. [CrossRef] [PubMed] 